\section{Severe symptomatic bradycardia after a dinner of spicy oleander soup}

\author{
Andrea Tampieri, Fabrizio Mucci, Valeria \\ Palmonari, Eugenio Giovannini, Tiziano \\ Lenzi, Patrizia Cenni \\ Emergency Department, S.M. Scaletta \\ Hospital, Imola (BO), Italy
}

\section{Abstract}

Cardiac glycosides similar to digoxin are produced by different plants in nature. Nerium oleander, commonly grown as an ornamental shrub, can be found worldwide in temperate countries. Intentional or accidental ingestion of any part of the plant can lead to clinically relevant intoxication. A 63-year-old woman came to the emergency department with acute dyspeptic symptoms after eating vegetable soup flavored with unfamiliar flowers she have collected herself. However, the electrocardiography (ECG) showed abnormalities that raised suspicions for an overdose of digoxin-like cardiac glycosides. The patient was not on treatment with digoxin and a careful anamnesis revealed that she had eaten oleander leaves. Digoxin specific Fab antibody fragments were administered for marked bradycardia that was not responding to atropine administration, after counseling with the reference toxicology center. The patient was also treated with activated charcoal and magnesium sulphate, intravenous fluids and pantoprazole. Four days later she was discharged as asymptomatic, with normal sinus rhythm. Emergency physicians should be aware of this type of poisoning, especially in cases with typical ECG alterations in patients not treated with digoxin and medical history of plants ingestion. Cardio-active glycosides are present in different plants, often used inappropriately, with potential toxic effects and harmful drug interactions.

\section{Introduction}

Digoxin-like cardiac glycosides are found in nature in a diverse group of plants including Digitalis purpurea and Digitalis lanata (foxgloves), Nerium oleander (common oleander) and Thevetia peruviana (yellow oleander). All cardiac glycosides bind to a site on the cell membrane, producing reversible inhibition of the sodium-potassium pump and subsequently increasing intracellular calcium concentrations in myocytes. Cardiac glycosides also have vagotonic effects that can result in bradycardia and heart block. Cardiovascular and gastrointestinal systems are primarily affected. Prolonged refractory period in atrioventricular (AV) node, shortened refractory periods and decreased resting membrane potential in myocytes may lead to potentially lethal arrhythmias such as sinus or AV block or refractory ventricular dysrhythmias. Nausea, vomiting and dyspeptic symptoms are also typical. Life threatening human toxicity may occur after ingestion of leaves, flowers, or seeds from plants containing cardiac glycosides. Intoxication by Nerium oleander (NO) is an uncommon event but occasional cases have been reported, and many of these exposures occurred in children and are usually unintentional. Mortality is rare but possible. We describe a symptomatic case of accidental oleander poisoning in an adult, diagnosed through electrocardiographic changes and a careful history collection and treated with digoxin specific FAB antibody fragments.

\section{Case Report}

A 63-year-old woman with a medical history of hypertension, diabetes and hypothyroidism came to the Emergency Department (ED) complaining of epigastric pain and vomiting that had started approximately 6 hours after a dinner of vegetable soup, and she was the only person who had eaten it. She was on treatment with beta blockers (bisoprolol $5 \mathrm{mg}$ once daily in the morning), oral antidiabetic (metformin $500 \mathrm{mg}$ o.d.), and levo-tiroxin (75 mcg in the morning). On arrival at the ED the patient was conscious with adequate perfusion. Her heart rate was of 60 beats/minute, blood pressure of $120 / 70 \mathrm{~mm} / \mathrm{hg}$, oxygen saturation of $96 \%$ in air and body temperature $36.9^{\circ} \mathrm{C}$. The abdomen was soft without signs of peritoneal irritation, and an abdominal ultrasound evaluation did not show gallstones nor other abnormalities. ECG on arrival (Figure 1) showed sinus rhythm with a markedly prolonged first degree AV block and a diffused ST depression with upper concavity. Reported symptoms and ECG alterations could be suggestive for myocardial ischemia (considering the cardiovascular risk factors involved), as well as for food-induced abdominal colic in patients with left ventricular hypertrophy, but also for intake of digoxin or digoxin-like substances although our patient was not on treatment with digoxin. A transthoracic echocardiogram was performed without detection of cardiac anomalies. Laboratory tests including complete blood count, hepatic, renal and thyroid function test, glycaemia, serum electrolytes, amylase, myocardial necrosis enzymes, and hemo-gas analysis were collected and resulted normal, while the serum digoxin level was $0.9 \mathrm{ng} / \mathrm{mL}$
Correspondence: Andrea Tampieri, Emergency Department, S.M. Scaletta Hospital, Imola (B0), Italy.

Tel: +39.0542.662711

E-mail: andrea.tampieri@libero.it

Key words: Plant poisoning; Nerium oleander; Cardiac glycosides; Digoxin antibodies FAB fragments; Cardiac dysrhythmia.

Received for publication: 30 August 2016. Accepted for publication: 6 September 2016.

This work is licensed under a Creative Commons Attribution 4.0 License (by-nc 4.0).

(C) Copyright A. Tampieri, et al., 2016

Licensee PAGEPress, Italy

Emergency Care Journal 2016; 12:6252

doi:10.4081/ecj.2016.6252

(our therapeutic range for digoxin is between 0.9 and $2 \mathrm{ng} / \mathrm{mL}$ ). A more careful anamnesis revealed that her dinner had been dressed with oleander leaves. The patient denied intention of self-harm and reported to have used $\mathrm{NO}$ as a garment in substitution of laurel, not being aware of its toxic potential. ECG modifications, suggestive also for digoxin-like cardiac glycosides intoxication, were probably due to NO poisoning. In our laboratory a specific method for oleandrin is not available and a Digoxin Flex reagent cartridge by Siemens is used for sampling digoxin with an immunoassay technique. This method presents only a limited cross reactivity for NO glycosides so the actual level of oleandrin or other digoxinlike cardiac glycosides was probably underestimated. After telephone counseling with the local toxicology center, the patient was treated with an oral administration of $50 \mathrm{~g}$ of activated charcoal together with $30 \mathrm{~g}$ of magnesium sulphate, intravenous fluids and $40 \mathrm{mg}$ of pantoprazole. The patient was subsequently admitted to the acute medicine ward for observation with ECG monitoring and treatment with repeated administration of activated charcoal ( $5 \mathrm{~g}$ every two hours for the first day and $5 \mathrm{~g}$ every six hours for 2 days). In the subsequent hours her cardiac rhythm varied between sinus rhythm with first-degree AV block, second-degree Mobitz type-I AV block (Figure 2) evolving into severe bradycardia (Figure 3) sustained by Mobitz type-II AV block not responding to atropine administration $(0.5 \mathrm{mg}$ i.v). Administration of $400 \mathrm{mg}$ of intravenous digoxin specific Fab antibody fragments was agreed on with the poison control center for the arrhythmic events observed. Six hours after Fab administration, the digoxin level was lower than our therapeutic range $(0.5 \mathrm{ng} / \mathrm{mL})$, the ECG improved with regression of the AV block from second to first degree and sinus rhythm was subsequently maintained. 
Abnormalities of repolarization (ST depression with the typical upper concavity) still persisted. Four days after admission, the patient was discharged in good health and electrocardiography abnormalities had regressed completely, as shown in Figure 4.

\section{Discussion}

Oleander is a native evergreen ornamental shrub in the Mediterranean area and is planted in many subtropical areas of the world. It is commonly used in landscaping motorway medians in mild-winter states and can also be found in colder countries, usually grown inside greenhouses. Flower colour varies from white to deep red. Every part of the plants, from root to flowers, contain many cardiac glycosides (of which oleandrin is the most studied and represented) that are structurally similar to digoxin. ${ }^{1}$ The highest concentration has been reported in the seeds, followed by the leaves and fruit. ${ }^{2}$ Even if it is commonly regarded as a poisonous plant, accidental ingestion of NO parts has been described both in adults and children. Other causes of intoxication include deliberate self-poisoning or ingestion of medicinal preparations. Intake of any part of the plant can cause symptoms similar to digoxin poisoning. Oral ingestion of 5 to 15 leaves of NO has resulted in fatal poisoning in adults ${ }^{3}$ and even one leaf has been suggested as potentially toxic for children. ${ }^{4}$ However, the exact lethal dose is hard to establish, as in many reported cases the specific ingested dose was not established. ${ }^{5}$ There is also a large variation among individuals in the amount of absorption of cardio-active toxins. Cardiac glycosides inactivate the $\mathrm{Na}+/ \mathrm{K}+$ ATPase pump on the cytoplasmic membrane of cardiac cells, raising the intracellular $\mathrm{Ca}++$ concentration and leading to inotropic effect, hyperkalaemia, and alterations of electrical impulse conduction. Symptoms of intoxication usually arise a few hours after ingestion. ${ }^{5}$ Drinking infusions made with leaves or roots is often associated with a faster onset compared to ingestion of unprepared plant parts. ${ }^{6,7}$ The typical clinical presentation includes gastrointestinal symptoms such as nausea, vomiting, abdominal pain, increased salivation or diarrhoea, together with cardiac dysrhythmias and hyperkalaemia. The more frequent cardiac dysrhythmias are conduction defects that affect the sinus or AV node, atrial fibrillation, ventricular ectopic beats or, less commonly, ventricular fibrillation. Neurological symptoms such as tremor, ataxia or visual alterations can be associated with oleander intoxication. A careful history collection (part of plant ingested, quantity, time since ingestion and symptoms) together with the ECG are important diagnos- tic elements for oleander poisoning.

In our specific case, gastrointestinal symptoms could be attributed to intoxication from a digoxin-like substance. Typical ECG alterations (progression of AV-block, diffuse ST depression with upper concavity, QTc interval shortened despite marked bradycardia in Figure 3) in a patient not treated with digoxin, together with a careful anamnesis, led to the diagnosis of NO poisoning. An interaction of cardiac glycosides with beta-blockers in the induction of bradycardia cannot be definitely excluded, however it seems unlikely considering that the half-life of bisoprolol (taken in the morning) is estimated about 10 hours, while the patient came to the ED later in the evening and it was immediately suspended. Whereas a specific test for NO cardiac glycosides is not available, the laboratory is of limited use, as many commonly-used tests for digoxin present only limited cross reactivity for other digoxin-like glycosides, which might prove to be undervalued. Despite the gastrointestinal decontamination performed 6 hours after NO ingestion, AVblock and bradycardia worsened during hospitalisation, suggesting an inappropriate timing in treatment. Atropine administration could have counteracted the vagotonic glycosides effect, but was not found to be effective on the treatment of bradycardia. Conversely, AV-block has regressed after Fab fragments infusion. Given the occasional nature of NO intoxication, there is no treatment supported by a strong evidence in the literature. Few randomised-controlled trials are actually available $^{8-10}$ and all of them addressed poisoning by Yellow Oleander (Thevetia peruviana) that contains slightly different cardiac glycosides from NO, commonly used for deliberate selfpoisoning in Sri Lanka. ${ }^{11}$ Actually, intoxication from common or yellow oleander is managed in the same way. Mainstays of treatment include supportive care with ECG monitoring, gastrointestinal decontamination and specific antidote administration.,12 Control of electrolytes and fluid balance is obviously essential in patients with vomiting or diarrhoea. Rapid correction of electrolyte alterations is of paramount importance to minimise cardiac arrhythmic complications. Marked bradycardia with hypotension can be treated with bolus doses of atropine or temporary pacing if antidigoxin Fab is not immediately available (even if in the case of extreme intoxication, cardiac cells with severe electric alterations can be refractory to external depolarisation). Gastroenteric decontamination is actually discussed. Repeated activated charcoal administration

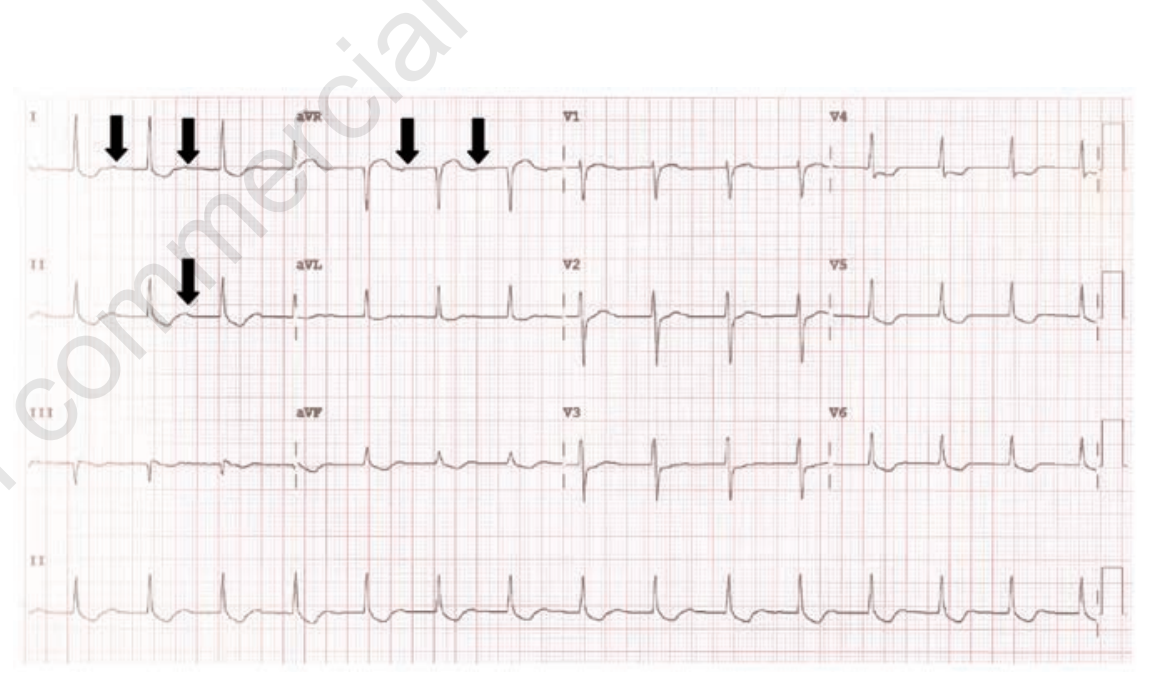

Figure 1. First electrocardiography on arrival showing sinus rhythm with first degree AV block (markedly prolonged PR interval with $P$ positive wave in DI and negative in aVR on the end of the T wave, and within the T wave in DII-black arrows) and a diffused ST depression with upper concavity.

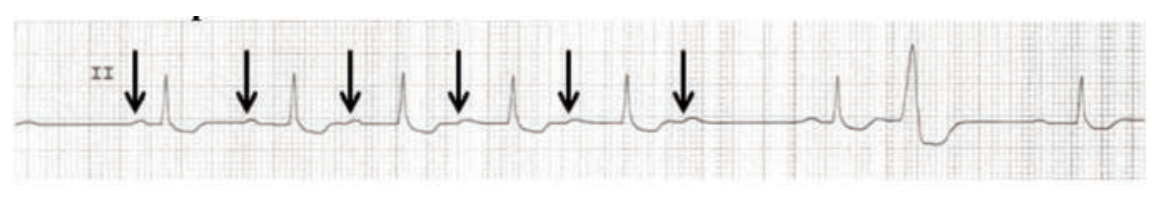

Figure 2. Electrocardiography monitoring of the second lead with second degree Mobitz type I AV block (progressively lengthened PR interval before a P wave not followed by any QRS complex) and an ectopic ventricular beat. 
has been studied in two randomised controlled trials with conflicting results. Silva et al. ${ }^{8}$ found multiple-dose activated charcoal administration (50 g every 6 hours for 3 days) effective in reducing deaths and life-threatening cardiac arrhythmias in patients admitted up to 24 hours after yellow oleander poisoning. A subsequent study by Eddleston et al. ${ }^{10}$ instead did not find significant changes in mortality related to charcoal administration either in single or in multiple doses. Given that treatment with activated charcoal was safe in both studies $^{8,10}$ and that it could increase the rate of elimination of cardiac glycosides, ${ }^{13-15}$ its use is generally recommended even if its effectiveness in reducing mortality is uncertain. Whole bowel irrigation has also been considered to reduce enteric absorption. ${ }^{4}$ Today, however, there is insufficient data to evaluate this treatment. As for specific antidotes for cardiac glycosides, polyclonal anti-digoxin Fab fragments are actually available and of clear benefit if administered for digoxin poisoning. ${ }^{16}$ These antibodies can also bind to oleandrin in vitro and reduce its active concentration. ${ }^{17} \mathrm{~A}$ randomised controlled trial by Eddleston et al. ${ }^{9}$ addressed the use of $1200 \mathrm{mg}$ of intravenous Fab antibodies for life-threatening cardiac arrhythmias in patients with yellow oleander poisoning, reporting a highly significant response to treatment. Alternatively, $400 \mathrm{mg}$ in
20 minutes followed by an infusion of $400-800$ mg over 6-8 hours has also been suggested. ${ }^{5}$ In case of advanced AV block, severe sinus node block and ventricular tachyarrhythmia, administration of anti-digoxin Fab is appropriate in patients with oleander poisoning, independently from serum digoxin concentration, as routinely used digoxin assays are at best semiquantitative for other digoxin-like substances. ${ }^{9,18,19}$ Hyperkalaemia (serum potassium $>5.5 \mathrm{mEq} / \mathrm{L}$ ) could also be an indication for Fab administration in oleander poisoning, as the antibodies decrease potassium concentrations significantly within 2 hours., ${ }^{9,18}$

\section{Conclusions}

NO is a plant commonly found in many countries worldwide. Cases of intoxication are uncommon but emergency physicians should be aware of this poisoning, especially in the case of typical ECG alterations in patients not treated with digoxin and with a medical history of plants ingestion. Both diagnosis and first treatment are often possible and can be managed by ED physicians. Principles of treatment are similar to those of digoxin intoxication. Electrolyte imbalances and cardiac arrhythmias should be corrected promptly. Gastric

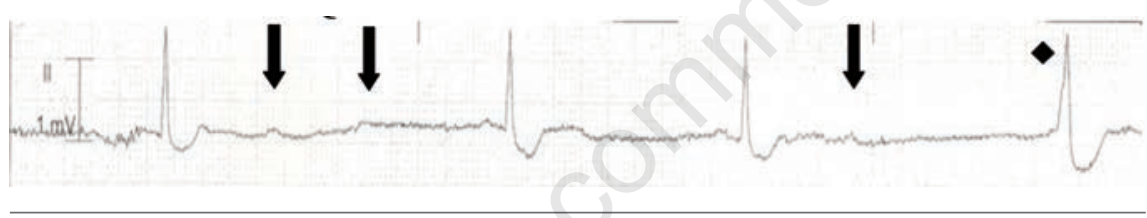

Figure 3. Electrocardiography monitoring: marked bradycardia (30 bpm) with non-conducted P wave (black arrows) as in Mobitz type II AV block, escape ventricular beat (black diamond), and mild shortened QTc interval.

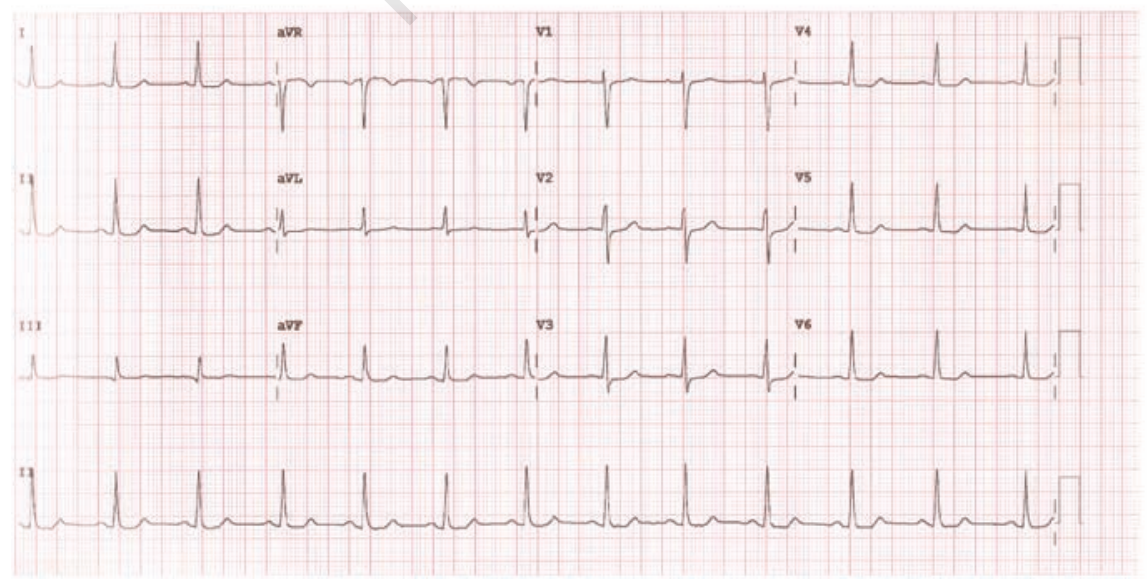

Figure 4. Electrocardiography at discharge showed a normal SE and a regression of STsegment abnormalities.

decontamination strategies must be evaluated and treatment with specific FAB antibodies is recommended in case of life-threatening arrhythmic events or severe hyperkalaemia. ECG monitoring should be maintained until complete resolution of the arrhythmic events.

\section{References}

1. Langford, SD, Boor PJ. Oleander toxicity: an examination of human and animal toxic exposures. Toxicology 1996;109:1-13.

2. Karawya MS, Balbaa SI, Khayyal SE. Estimation of cardenolides in Nerium oleander. Planta Med 1973;23:70-3.

3. Osterloh J, Herold S, Pond S. Oleander interference in the digoxin radioimmunoassay in a fatal ingestion. J Am Med Assoc 1982;247:1596-7.

4. Shaw D, Pearn J. Oleander poisoning. Med J Aust 1979;8:267-9.

5. Bandara V, Weinstein SA, White J, Eddleston M. A review of the natural history, toxicology, diagnosis and clinical management of Nerium oleander (common oleander) and Thevetia peruviana (yellow oleander) poisoning. Toxicon 2010;56:27381.

6. Haynes BE, Bessen HA. Oleander tea: herbal draught of death. Ann Emerg Med 1985;14:350-3.

7. Le Couteur DG, Fisher AA. Chronic and criminal administration of Nerium oleander. J Toxicol Clin Toxicol 2002;40:523-4.

8. de Silva HA, Fonseka MMD, Pathmeswaran A, et al. Multiple-dose activated charcoal for treatment of yellow oleander poisoning: a single-blind, randomised, placebo-controlled trial. Lancet 2003;361:1935-8.

9. Eddleston M, Rajapakse S, Rajakanthan K, et al. Anti-digoxin Fab fragments in cardiotoxicity induced by ingestion of yellow oleander: a randomised controlled trial. Lancet 2000;355:967-72.

10. Eddleston M, Juszczak E, Buckley NA, et al. Multiple-dose activated charcoal in acute self-poisoning: a randomised controlled trial. Lancet 2008;371;579-87.

11. Eddleston M, Sheriff MHR, Hawton K. Deliberate self harm in Sri Lanka: an overlooked tragedy in the developing world. Brit Med J 1998;317:133-5.

12. Rajapakse S. Management of yellow oleander poisoning. J Toxicol Clin Toxicol 2009;47:206-12.

13. Chyka PA, Holley JE, Mandrell TD, et al. Correlation of drug pharmacokinetics and effectiveness of multiple-dose activated charcoal therapy. Ann Emerg Med 1995; 25:356-62.

14. Lalonde RL, Deshpande R, Hamilton PP, et 
al. Acceleration of digoxin clearance by activated charcoal. Clin Pharmacol Ther 1985;37:367-71.

15. Ibanez C, Carcas AJ, Frias J, Abad F. Activated charcoal increases digoxin elimination in patients. Int $\mathrm{J}$ Cardiol 1995; 48:27-30.

16. Antman EM, Wenger TL, Butler VP, et al. Treatment of 150 cases of life-threatening digitalis intoxication with digoxin-specific
Fab antibody fragments: final report of a multicenter study. Circulation 1990;81:1744-52.

17. Dasgupta A, Hart AP. Rapid detection of oleander poisoning using fluorescence polarization immunoassay for digitoxin. Effect of treatment with digoxin-specific Fab antibody fragment (ovine). Am J Clin Pathol 1997;108:411-6.

18. Eddleston M, Ariaratnam CA, Sjostrom L, et al. Acute yellow oleander (Thevetia peruviana) poisoning: cardiac arrhythmias, electrolyte disturbances, and serum cardiac glycoside concentrations on presentation to hospital. Heart 2000;83:301-6.

19. Osterloh J, Harold S, Pond S. Oleander interference in the digoxin radioimmunoassay in a fatal ingestion. J Am Med Assoc 1982;247:1596-7. 